\title{
Multi-messenger constraints to the local emission of cosmic-ray electrons
}

\author{
Fiorenza Donato* \\ Dipartimento di Fisica, Università di Torino, Via P. Giuria 1, 10125 Torino, Italy \\ Istituto Nazionale di Fisica Nucleare, Sezione di Torino, Via P. Giuria 1, 10125 Torino, Italy \\ E-mail: donato@to.infn.it
}

\section{Silvia Manconi}

Dipartimento di Fisica, Università di Torino, Via P. Giuria 1, 10125 Torino, Italy

Istituto Nazionale di Fisica Nucleare, Sezione di Torino, Via P. Giuria 1, 10125 Torino, Italy

E-mail: manconi@to.infn.it

\section{Mattia di Mauro}

NASA Goddard Space Flight Center, Greenbelt, MD 20771, USA

Catholic University of America, Department of Physics, Washington DC 20064, USA

\begin{abstract}
The data on the inclusive flux of cosmic positrons and electrons $\left(e^{+}+e^{-}\right)$have been recently collected from $\mathrm{GeV}$ to tens of $\mathrm{TeV}$ energies by several experiments with unprecedented precision. In addition, the Fermi-LAT Collaboration has provided a new energy spectrum for the upper bounds on the $e^{+}+e^{-}$dipole anisotropy. This observable can bring information on the emission from local Galactic sources, notably measured with high precision at radio frequencies. We develop a framework in which $e^{-}$and $e^{+}$measured at Earth from $\mathrm{GeV}$ up to tens of TeV energies have a composite origin. A dedicated analysis is deserved to Vela $\mathrm{YZ}$ and Cygnus Loop Supernova Remnants (SNRs), for which we consider two different models for the injection of $e^{-}$. We investigate the consistency of these models using the three physical observables: the radio flux from Vela $\mathrm{YZ}$ and Cygnus Loop at all the available frequencies, the $e^{+}+e^{-}$flux from five experiments from the $\mathrm{GeV}$ to tens of $\mathrm{TeV}$ energy, the $e^{+}+e^{-}$dipole anisotropy upper limits from $50 \mathrm{GeV}$ to about $1 \mathrm{TeV}$. We find that the radio flux for these nearby SNRs strongly constraints the properties of the injection electron spectrum, partially compatible with the looser constraints derived from the $e^{+}+e^{-}$flux data. We also perform a multi-wavelength multi-messenger analysis by fitting simultaneously the radio flux on Vela $\mathrm{YZ}$ and Cygnus Loop and the $e^{+}+e^{-}$flux, and checking the outputs against the $e^{+}+e^{-}$dipole anisotropy data. Remarkably, we find a model which is compatible with all the $e^{+}+e^{-}$flux data, the radio data for Vela YZ and Cygnus Loop, and with the anisotropy upper bounds. We show the severe constraints imposed by the most recent data on the $e^{+}+e^{-}$dipole anisotropy.
\end{abstract}

36th International Cosmic Ray Conference -ICRC2019-

July 24th - August 1st, 2019

Madison, WI, U.S.A.

\footnotetext{
* Speaker.
} 


\section{Introduction}

The flux of cosmic-ray (CR) electrons and positrons $\left(e^{-}\right.$and $\left.e^{+}\right)$has been measured with unprecedented precision over more than four orders of magnitude of energy. One of the most accurate measurements on single $\mathrm{CR} e^{-}$and $e^{+}$and inclusive $\left(e^{+}+e^{-}\right)$fluxes is provided by AMS-02 on board the International Space Station (ISS), between $0.1 \mathrm{GeV}$ to $1 \mathrm{TeV}$ energy, and with errors reaching the few percent level $[1,2,3]$. The Fermi Large Area Telescope (LAT) has collected almost seven years of $e^{+}+e^{-}$events in the $7 \mathrm{GeV}-2 \mathrm{TeV}$ energy range [4]. CALET on the ISS, and HESS on the ground, are providing $e^{+}+e^{-}$data up to $3 \mathrm{TeV}$ and $30 \mathrm{TeV}$ energy, respectively $[5,6,7]$. The DAMPE Collaboration has recently reported the direct detection of a break at around $1 \mathrm{TeV}$ in the flux of the $e^{+}+e^{-}$measured between $25 \mathrm{GeV}$ to $4.6 \mathrm{TeV}$ [8]. In addition to the flux, the LAT team has also published the spectrum of upper limits on the $e^{+}+e^{-}$ dipole anisotropy [9]. Since the typical propagation length of $\mathrm{TeV} e^{ \pm}$is smaller than $\sim 0.3 \mathrm{kpc}, e^{+}$ and $e^{-}$detected at $\mathrm{TeV}$ energies are most probably emitted from local sources, leaving a possible signature in the dipole anisotropy [10].

The contribution from the local source candidates is usually associated with high uncertainties, primarily connected to the properties of the accelerated and emitted $e^{-}$and $e^{+}$. Moreover, the completeness of current catalogs, such as SNRs, is assessed by means of the observed surface brightness (see e.g. [11]), thus leaving open the possibility that nearby and very old sources may contribute to the flux at Earth even if they are no longer visible at any energy of the electromagnetic band. A strategy to constrain the source contributions of local known sources is to model their multi-wavelength emission and to connect it to the emitted CRs. For example, the lepton emission from sources embedded in a magnetic field, such as $e^{-}$from SNRs, can be connected with their synchrotron emission at radio frequencies (see $[12,13,10]$ and references therein). In addition, the most recent experimental upper bounds on the dipole anisotropy could set further limits on the properties of local and dominant sources.

We use this strategy to quantify the contribution of local known sources, in particular from two SNRs which are widely considered as the main candidates to contribute significantly to the high energy part of the $e^{-}$flux at Earth (often measured cumulatively through $e^{-}+e^{+}$), namely Vela and Cygnus Loop, see e.g. [14]. We present here a multi-component model that explains the $e^{+}$ and $e^{-}+e^{+}$fluxes from five experiments and in a wide energy range, and that is simultaneously compatible with the upper bounds on the dipole anisotropy and the radio emission from the most intense and closest SNRs [15].

\section{Electron and positrons at the Earth}

Under the hypothesis that the radio emission from the SNR is due to synchrotron radiation from $e^{-}$accelerated and interacting with the SNR magnetic field $B$, the normalization of the injection spectrum $Q_{0, \mathrm{SNR}}$ can be connected to the radio flux density $B_{r}^{v}(v)$ :

$$
Q_{0, \mathrm{SNR}}=1.2 \cdot 10^{47} \mathrm{GeV}^{-1}(0.79)^{\gamma} \frac{B_{r}^{v}(v)}{\mathrm{Jy}}\left[\frac{d}{\mathrm{kpc}}\right]^{2}\left[\frac{v}{\mathrm{GHz}}\right]^{\frac{\gamma-1}{2}}\left[\frac{B}{100 \mu \mathrm{G}}\right]^{-\frac{\gamma+1}{2}} .
$$

The derivation of this expression is extensively provided in [12], and it was successively used also in $[13,10]$. As for the treatment of the propagation in the Galaxy and the energy losses suffered by 
electrons from the source to the Earth we refer to $[15,10]$.

In Fig. 1 we compute the flux of the seven SNRs which are located at $d<1 \mathrm{kpc}$ from the Earth in the Green SNR catalog [16]. Vela YZ turns out to be the most powerful source, followed by Cygnus Loop. Electrons from the other sources have fluxes smaller than up one order of magnitude. We identify Vela YZ and Cygnus Loop as the candidates expected to contribute most significantly to the high-energy tail of $e^{+}+e^{-}$flux, given their distance, age and radio flux [14, 13, 10]. With respect to previous analysis where usually a single frequency was considered (see, e.g., $[13,17])$, we use here the radio spectrum in the widest available range of frequencies. The parameter space $E_{\text {tot }}-\gamma$ selected by the fit to the radio spectrum is reported in the left panel of Fig. 2 for both Vela $\mathrm{YZ}$ and Cygnus Loop, and for $3 \sigma, 2 \sigma$ and $1 \sigma$ confidence levels. This figure shows that radio data select narrow ranges for $\gamma$ and $E_{\text {tot }}$. In the right panel of Fig. 2 we plot the data on the $e^{+}+e^{-}$flux along with the predictions for the flux from Vela YZ and Cygnus Loop obtained by the parameters selected within the $2 \sigma$ contours in the left panel. We address to [15] for further details.

In addition to the burst-like approximation, we also implement the evolutionary model for the escape of $e^{-}$from SNRs as derived in Ref. [18] and detailed in [15]. We have explored the effects of the evolutionary escape model on the interpretation of radio spectrum for our two selected sources. In Fig. 3 (left panel) the parameter space $E_{\text {tot,trap }}-\gamma\left(E_{\text {tot,esc }}-\gamma+\beta / \alpha\right)$ selected by the fit to the radio spectrum of Vela YZ and Cygnus Loop are shown for $3 \sigma, 2 \sigma$ and $1 \sigma$ confidence levels. The selected intervals are narrow, and similar to the burst-like case for Vela YZ. The derived constraints on the parameter space $E_{\text {totesc }}-\gamma+\beta / \alpha$ are also reported. Each of the two regions shows a strong correlation. The consequences of these results on the $e^{+}+e^{-}$flux are reported in Fig. 3 (right panel). We plot again the data on the $e^{+}+e^{-}$flux, along with the predictions for the flux of runaway $e^{-}$from Vela YZ and Cygnus Loop. Considering all the uncertainties, under the evolutionary escape model the flux from Vela YZ and Cygnus Loop is predicted to contribute at most few percent to the data on the inclusive flux at $\mathrm{TeV}$ energies

We then compute the dipole anisotropy [10] for Vela YZ and Cygnus Loop for all the parameters selected by the fit to $e^{+}+e^{-}$flux data as performed in [15]. The maximum of $\Delta_{e^{+}+e^{-}}$in each energy bin is then plotted as a black (magenta) solid line in Fig. 4 for Vela YZ (Cygnus Loop). For
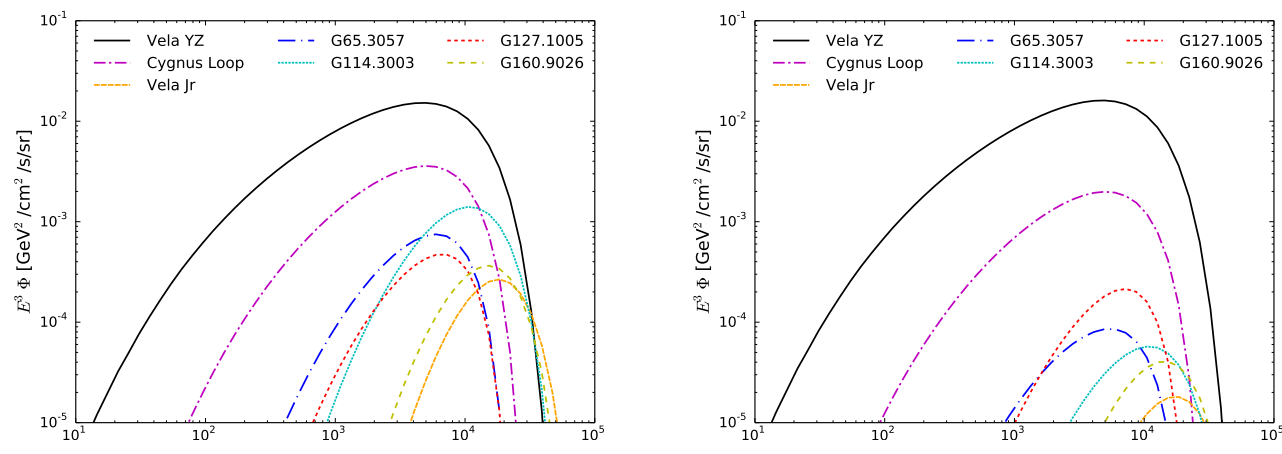

Figure 1: Electron flux at Earth from near SNRs in the Green catalog at $d<1 \mathrm{kpc}$ from the Earth. Left: A common spectral index of $\gamma=2.0$ and a total energy released in $e^{-}$of $E_{\text {tot }}=7 \cdot 10^{47} \mathrm{erg}$ has been assumed for each source. Right: The spectral index and the $Q_{0}$ for each source are fixed according to the catalog data. All the curves are computed for $E_{c}=10 \mathrm{TeV}$ and $\mathrm{K} 15$ propagation model. 

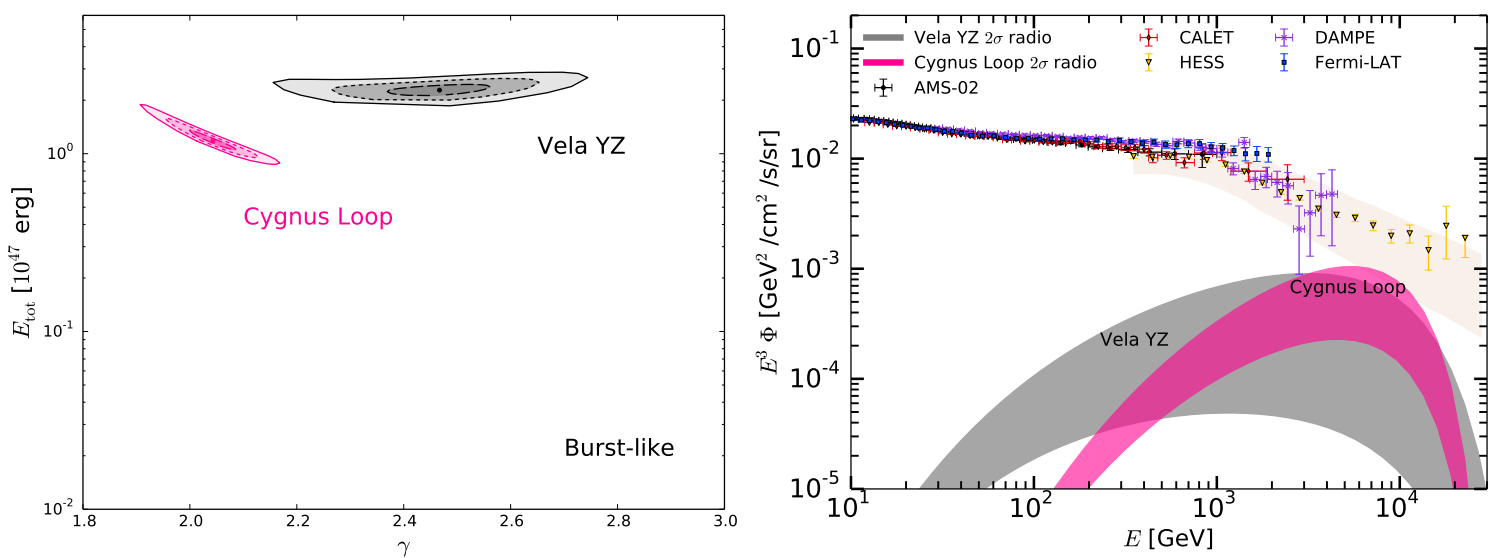

Figure 2: Results of the fit to the radio spectrum for Vela YZ (gray) and Cygnus Loop (magenta). Left: Regions of the parameter space $E_{\text {tot }}, \gamma$ selected by the fit to the radio spectrum. The solid, dashed and longdashed lines refer to respectively $3 \sigma, 2 \sigma$ and $1 \sigma$ contours for each source. Right: Prediction for the $e^{-}$flux from Vela $\mathrm{YZ}$ and Cygnus Loop using the values of $E_{\mathrm{tot}}, \gamma$ within $2 \sigma$ from the best fit to the radio spectrum shown in the left panel. The $e^{+}+e^{-}$Fermi-LAT, AMS-02, DAMPE, HESS and CALET data with their statistics and systematic errors are also shown.

Vela YZ, the anisotropy overshoots Fermi-LAT [9]. upper limits on the whole spectrum. We can therefore infer that Fermi-LAT data on the lepton dipole anisotropy add an independent piece of information in addition to the flux data. We then compute $\Delta_{e^{+}+e^{-}}$for all the configurations selected by the fit to the flux. Whenever our predictions overestimate one data point at $E>100 \mathrm{GeV}$, the $E_{\text {tot,Vela }}-\gamma_{\text {Vela }}$ pair is considered as excluded. The results are displayed by the hatched region in the right panel of Fig. 4. The dipole anisotropy upper limits are not compatible with the configurations selected by the fit to the flux data at $2 \sigma$, and with a subset of the configurations at $5 \sigma$. The Fermi-LAT data on $\Delta_{e^{+}+e^{-}}$supplement a valuable information of the properties of Vela YZ, acting as a further physical observable for the understanding of the injection of $e^{-}$in the ISM.

We now combine all the three observables explored previously. Specifically, we compare the dipole anisotropy of Vela YZ and Cygnus Loop with the Fermi-LAT upper bounds, for the parameters of these sources selected by radio and $e^{+}+e^{-}$fluxes. We perform new fits on the $e^{+}+e^{-}$ and $e^{+}$fluxes including the constraints for $E_{\text {tot, Vela }}, \gamma_{\text {Vela }}, E_{\text {tot,Cygnus }}$, and $\gamma_{\text {Cygnus }}$ derived from the fit to radio data. We find a very good agreement between $e^{+}+e^{-}$and radio data $\left(\chi_{\text {red }}^{2} \approx 0.70\right)$ with $\gamma_{\text {Vela }}=2.39 \pm 0.15, E_{\text {tot,Vela }}=(2.3 \cdot \pm 0.2) \cdot 10^{47} \mathrm{erg}, \gamma_{\text {Cygnus }}=2.03 \pm 0.05$ and $E_{\text {tot,Cygnus }}=$ $(1.25 \pm 0.06) \cdot 10^{47} \mathrm{erg}$ for K15 (see [15] for details) propagation models. We illustrate in Fig. 5 the result of the best fit for all the components to the $e^{+}+e^{-}$flux. We checked that all the predictions for the dipole anisotropy within $2 \sigma$ from the best fit are below the Fermi-LAT upper bounds, as explicitly shown in Fig. 5, right panle. The putative $e^{-}$injected by a radio unconstrained Vela SNR are compensated in our framework by the combination of $e^{-}$produced by the Galactic smooth distribution of SNRs and all the PWNe. Also in the evolutionary model, we find a good fit. The parameters which describes the smooth SNRs, the PWNe and the secondary component are compatible within the errors with respect to the burst-like scenario. We refer the interested reader to [15] for any further detail of the present analysis. 

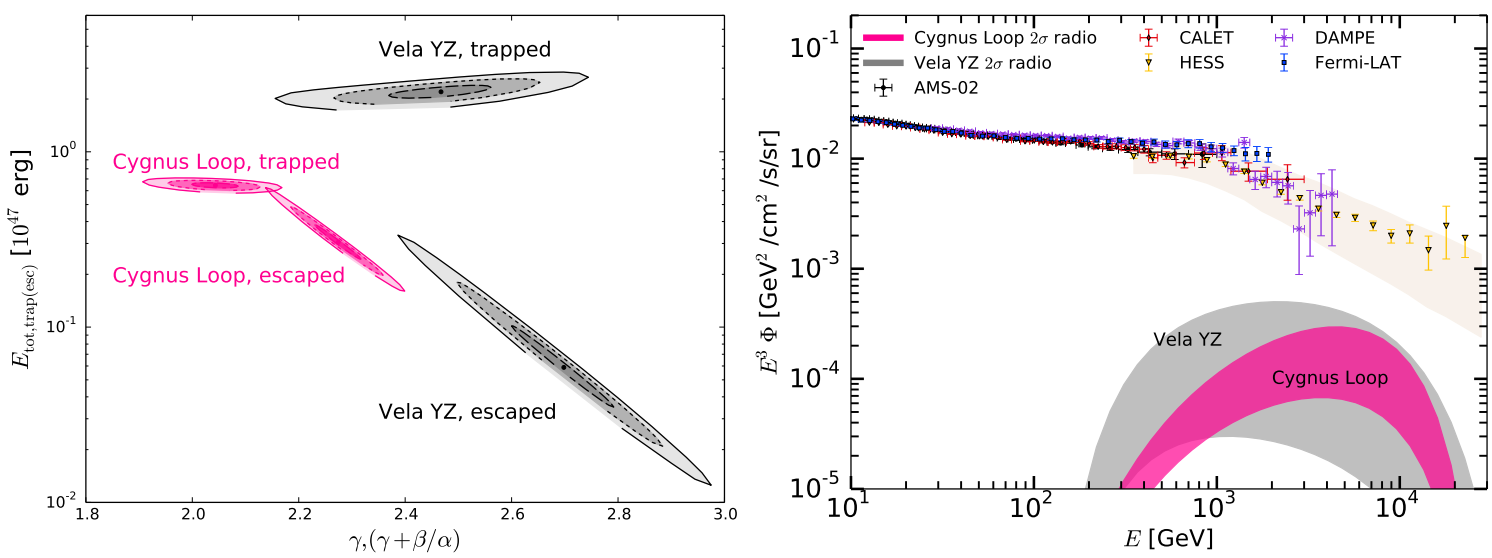

Figure 3: Results of the fit to the radio spectrum for Vela YZ (gray) and Cygnus Loop (magenta) for the evolutionary model of the injection of $e^{-}$from SNRs in Ref. [18]. Left: Regions of the parameter space $E_{\text {tot,trap }}, \gamma$ selected by the fit to the radio spectrum for Vela YZ (gray) and Cygnus Loop (magenta). The derived regions for $E_{\mathrm{totesc}}, \gamma+\beta / \alpha$ are also reported for Vela $\mathrm{YZ}$ and Cygnus Loop. The solid, dashed and long-dashed lines refer to respectively $3 \sigma, 2 \sigma$ and $1 \sigma$ contours for each source. Right: Prediction for the $e^{-}$ flux from Vela YZ and Cygnus Loop using the values of $E_{\text {tot,esc }}, \gamma+\beta / \alpha$ within $2 \sigma$ from the best fit to the radio spectrum shown in the left panel.

\section{Conclusions}

We investigate the compatibility of two different models for the emission and propagation of $e^{-}$and $e^{+}$in the Galaxy using three physical observables:

- the radio flux at all the available frequencies from Vela YZ and Cygnus Loop SNRs,

- the $e^{+}+e^{-}$flux from five experiments from the $\mathrm{GeV}$ to tens of $\mathrm{TeV}$ energy,

- the $e^{+}+e^{-}$dipole anisotropy upper limits from $50 \mathrm{GeV}$ to about $1 \mathrm{TeV}$.

We find that the radio flux for these nearby SNRs strongly constraints the total energy and the spectral index of the emitted $e^{-}$. In the case of the evolutionary escape model, we derive constraints on the total energy and spectral index of both trapped and runaway $e^{-}$. As for the burst-like approximation, the flux of $e^{-}$from Vela YZ and Cygnus Loop as derived from a fit to radio data is slightly below the data on the inclusive flux. It can skim the HESS data, when all the uncertainties are considered. In the assumption that all the radio emission is synchrotron radiation from $e^{-}$, our predictions indicate the highest flux expected from these sources can shape the high energy tail of the $e^{+}+e^{-}$flux data. In the case of the evolutionary escape model, the flux of runaway $e^{-}$ from Vela YZ and Cygnus Loop is slightly lower, and their contribution to the $e^{+}+e^{-}$flux data is subdominant with respect to the other model components.

We perform a radio-blind analysis by fitting only and all the most recent $e^{+}+e^{-} f l u x$ data. The data select correlated values for the total energy and spectral index of Vela YZ, and to a less extent of Cygnus Loop. The results for Vela YZ are compatible with the radio analysis within errors considered at $5 \sigma$ confidence level. 

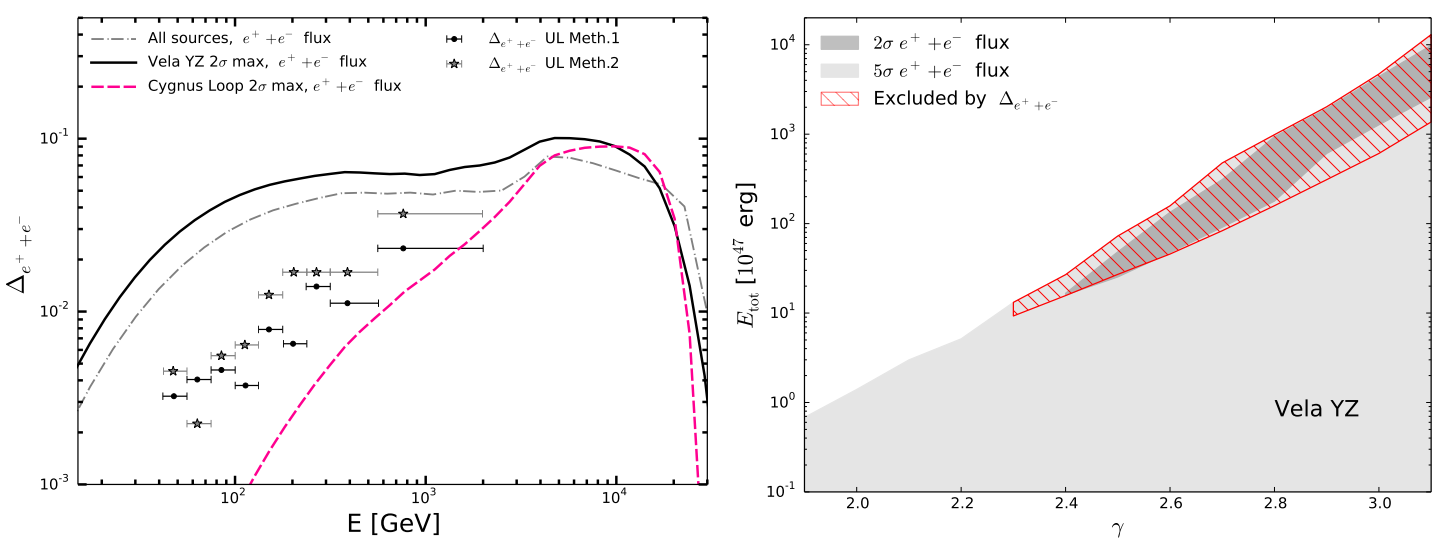

Figure 4: Left: dipole anisotropy predictions for Vela $\mathrm{YZ}$ and Cygnus Loop treated as single dominant sources (solid black and magenta lines, respectively), and for all the sources combined together, shown as gray dot-dashed line (see text for details). The upper limits for Fermi-LAT dipole anisotropy are shown. Right: Dipole anisotropy constraints to the Vela YZ source parameters. The regions of the parameter space $E_{\mathrm{tot}}, \gamma$ selected by the fit to the $e^{+}+e^{-}$and $e^{+}$flux data for Vela $\mathrm{YZ}$ are reported with shaded regions. The hatched region denotes the configurations selected by $e^{+}+e^{-}$and $e^{+}$flux data and excluded by Fermi-LAT dipole anisotropy upper limits at $E>100 \mathrm{GeV}$.
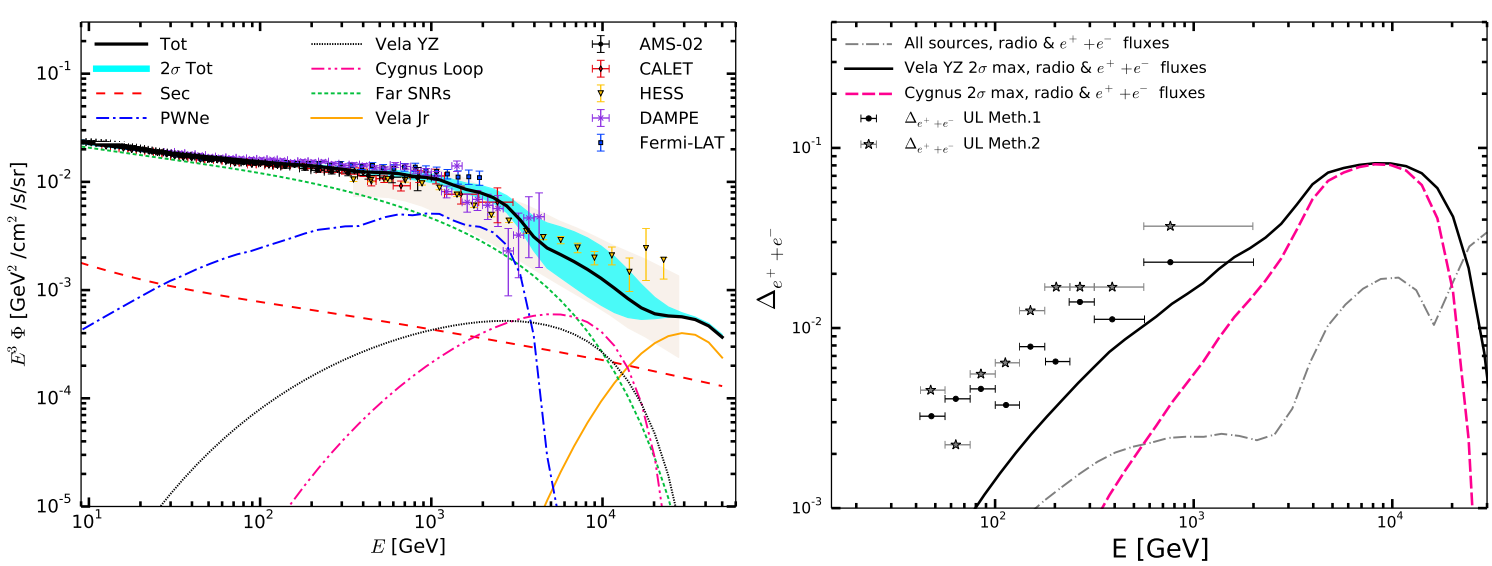

Figure 5: Results on the $e^{+}+e^{-}$flux (left) and on the corresponding dipole anisotropies (right) from the multi-wavelength fit to all the data. Left: The contribution from secondary production (red dashed), PWNe (blue dot dashed), Vela YZ (black dotted), Cygnus Loop (magenta dot-dot dashed), Vela Jr (orange solid) and the far smooth distribution of SNRs (green dotted) are shown. The $e^{+}+e^{-}$Fermi-LAT, AMS-02, DAMPE, HESS and CALET data with their statistics and systematic errors are also shown. Right: The maximal dipole anisotropy predicted for Vela YZ and Cygnus Loop as single dominant sources are reported with black solid and magenta dashed lines. The total anisotropy resulting from the distribution of all the sources is shown with gray dot-dashed line. The upper limits for Fermi-LAT dipole anisotropy are shown. 
As a further novelty, we consider the upper limits on $e^{+}+e^{-}$dipole anisotropy as an additional observable, and assess its power in constraining the Vela $\mathrm{YZ}$ and Cygnus Loop source properties. This operation is performed at the cost of no new free parameters. We find that the anisotropy overshoots Fermi-LAT upper limits on the whole spectrum when the Vela SNR parameters are left free to fit the $e^{+}+e^{-}$flux data. For Cygnus Loop the conclusions are weaker, since it shines at higher energies where the Fermi-LAT upper bounds are looser. The results are very similar when all the single sources considered in the analysis (SNRs and PWNe) contribute to the anisotropy, which is dominated by Vela YZ. We show the severe constraints imposed by the most recent data on the $e^{+}+e^{-}$anisotropy, what opens the opportunity of describing the most promising local sources of $e^{-}$with charged lepton CRs.

We finally perform a multi-wavelength multi-messenger analysis by fitting simultaneously the radio flux on Vela YZ and Cygnus Loop and the $e^{+}+e^{-}$flux, and checking the outputs against the $e^{+}+e^{-}$dipole anisotropy data. Considering the proper systematic uncertainties on the energy scale of the different data sets, we can fit the $e^{+}+e^{-}$spectrum on many energy decades using these local SNRs, a smooth distribution of SNRs, PWNe and secondary production. Remarkably, we find a model which is compatible with all the $e^{+}+e^{-}$flux data, the radio data for Vela YZ and Cygnus Loop, and with the anisotropy upper bounds.

\section{References}

[1] AMS collaboration, High Statistics Measurement of the Positron Fraction in Primary Cosmic Rays of 0.5-500 GeV with the Alpha Magnetic Spectrometer on the International Space Station, Phy. Rev. Lett. 113 (2014) 121101.

[2] AMS collaboration, Electron and Positron Fluxes in Primary Cosmic Rays Measured with the Alpha Magnetic Spectrometer on the International Space Station, Phy. Rev. Lett. 113 (2014) 121102.

[3] AMS collaboration, Precision Measurement of the $\left(e^{+}+e\right)$ Flux in Primary Cosmic Rays from 0.5 $\mathrm{GeV}$ to $1 \mathrm{TeV}$ with the Alpha Magnetic Spectrometer on the International Space Station, Phy. Rev. Lett. 113 (2014) 221102.

[4] FERMI-LAT collaboration, Cosmic-ray electron-positron spectrum from $7 \mathrm{GeV}$ to $2 \mathrm{TeV}$ with the Fermi Large Area Telescope, Phy. Rev. D 95 (2017) 082007.

[5] CALET collaboration, Energy spectrum of cosmic-ray electron and positron from $10 \mathrm{gev}$ to 3 tev observed with the calorimetric electron telescope on the international space station, Phy. Rev. Lett. 119 (2017) 181101.

[6] HESS collaboration, The cosmic-ray electron spectrum measured with h.e.s.s, talk at International Cosmic Ray Conference [CRI215], (2017) .

[7] O. Adriani et al., Extended Measurement of the Cosmic-Ray Electron and Positron Spectrum from 11 $\mathrm{GeV}$ to $4.8 \mathrm{TeV}$ with the Calorimetric Electron Telescope on the International Space Station, Phys. Rev. Lett. 120 (2018) 261102 [1806.09728].

[8] DAMPE collaboration, Direct detection of a break in the teraelectronvolt cosmic-ray spectrum of electrons and positrons, Nature 552 (2017) 63 [1711.10981].

[9] Fermi-LAT collaboration, Search for Cosmic-Ray Electron and Positron Anisotropies with Seven Years of Fermi Large Area Telescope Data, Phy. Rev. Lett. 118 (2017) 091103 [1703. 01073]. 
[10] S. Manconi, M. Di Mauro and F. Donato, Dipole anisotropy in cosmic electrons and positrons: inspection on local sources, JCAP 1701 (2017) 006 [1611.06237].

[11] D. A. Green, Constraints on the distribution of supernova remnants with Galactocentric radius, MNRAS 454 (2015) 1517 [1508.02931].

[12] T. Delahaye, J. Lavalle, R. Lineros, F. Donato and N. Fornengo, Galactic electrons and positrons at the Earth: new estimate of the primary and secondary fluxes, A\&A 524 (2010) A51 [1002.1910].

[13] M. Di Mauro, F. Donato, N. Fornengo et al., Interpretation of AMS-02 electrons and positrons data, JCAP 1404 (2014) 006 [1402.0321].

[14] T. Kobayashi, Y. Komori, K. Yoshida and J. Nishimura, The most likely sources of high energy cosmic-ray electrons in supernova remnants, ApJ 601 (2004) 340 [a stro-ph/0308470].

[15] S. Manconi, M. Di Mauro and F. Donato, Multi-messenger constraints to the local emission of cosmic-ray electrons, JCAP 1904 (2019) 024 [1803. 01009 ].

[16] D. Green, A catalogue of 294 Galactic supernova remnants, Bull.Astron.Soc.India 42 (2014) 47 [1409.0637].

[17] M. Di Mauro et al., Theoretical interpretation of Pass 8 Fermi-LAT $e^{+}+e^{-}$data, ApJ 845 (2017) 107 [1703.00460].

[18] Y. Ohira, R. Yamazaki, N. Kawanaka and K. Ioka, Escape of cosmic-ray electrons from supernova remnants, MNRAS 427 (2012) 91 [1106.1810]. 